GA-A24466

\title{
RECENT DEVELOPMENTS IN ALTERNATIVES TO CAMAC FOR DATA ACQUISITION AT DIII-D
}

\author{
by \\ D.H. KELLMAN, G.L. CAMPBELL, J.R. FERRON, \\ D.A. PIGLOWSKI, M.E. AUSTIN, and G.R. MCKEE
}




\section{DISCLAIMER}

This report was prepared as an account of work sponsored by an agency of the United States Government. Neither the United States Government nor any agency thereof, nor any of their employees, makes any warranty, express or implied, or assumes any legal liability or responsibility for the accuracy, completeness, or usefulness of any information, apparatus, product, or process disclosed, or represents that its use would not infringe privately owned rights. Reference herein to any specific commercial product, process, or service by trade name, trademark, manufacturer, or otherwise, does not necessarily constitute or imply its endorsement, recommendation, or favoring by the United States Government or any agency thereof. The views and opinions of authors expressed herein do not necessarily state or reflect those of the United States Government or any agency thereof. 


\title{
RECENT DEVELOPMENTS IN ALTERNATIVES TO CAMAC FOR DATA ACQUISITION AT DIII-D
}

\author{
by \\ D.H. KELLMAN, G.L. CAMPBELL, J.R. FERRON, \\ D.A. PIGLOWSKI, M.E. AUSTIN, $\dagger$ and G.R. MCKEE
}

\author{
tUniversity of Texas, Austin \\ fUniversity of Wisconsin, Madison
}

This is a preprint of a paper presented at the 20th IEEE/NPSS Symposium on Fusion Engineering, San Diego, California, October 14-17, 2003 and to be published in Fusion Science and Technology.

\author{
Work supported by \\ the U.S. Department of Energy \\ under Contract No. DE-AC03-99ER54463, and Grant Nos. \\ DE-FG03-97-ER54414, and DE-FG02-89ER53296
}




\title{
Recent Developments In Alternatives To CAMAC For Data Acquisition At DIII-D
}

\author{
D.H. Kellman, ${ }^{*}$ G.L. Campbell, ${ }^{*}$ J.R. Ferron, ${ }^{*}$ D.A. Piglowski, ${ }^{*}$ M.E. Austin,${ }^{\dagger}$ and G.R. McKee $\ddagger$ \\ ${ }^{*}$ General Atomics, P.O. Box 85608, San Diego, California 92186-5608 \\ tUniversity of Texas, Austin, Texas \\ †University of Wisconsin, Madison, Wisconsin
}

\begin{abstract}
For over twenty years, data acquisition hardware at DIII-D has been based on the CAMAC platform. These rugged and reliable systems, however, are gradually becoming obsolete due to end-of-life issues, ever-decreasing industry support of older hardware, and the availability of modern alternative hardware with superior performance. Efforts are underway at DIII-D to adopt new data acquisition solutions which exploit modern technologies and surpass the limitations of the CAMAC standard. These efforts have involved the procurement and development of data acquisition systems based on the PCI and Compact-PCI platform standards. These systems are comprised of rack-mount computers containing data acquisition boards ("digitizers"), Ethernet connectivity, and the drivers and software necessary for control. Each digitizer contains analog-to-digital converters, control circuitry, firmware and memory to collect, store, and transfer waveform data acquired using internal or external triggers and clocks. Software has been developed which allows DIII-D computers to program the operational parameters of the digitizers, as well as to upload acquired data into the DIII-D acquisition database. All communication between host computers and the new acquisition systems occurs via standard Ethernet connections, a vast improvement over the slower, serial loop highways used for control and data transfer with CAMAC systems. In addition, the capabilities available in modern integrated and printed circuit manufacture result in digitizers with high channel count and memory density. Cost savings are also realized by utilizing a platform based on standards of the personal computer industry. Details of the new systems at DIII-D are presented, along with initial experience with their use, and plans for future expansion and improvement.
\end{abstract}

\section{INTRODUCTION}

The acquisition of experimental data at DIII-D is predominately accomplished through the use of CAMAC systems. With these systems, data acquisition modules ("digitizers") and memory modules are installed in enclosures ("crates") which reside throughout the facility in proximity to sources of data. Crates are interconnected via CAMAC "highways", which are essentially serial loop networks. A host computer communicates with the crates by means of a serial highway driver connected to the highway.

The CAMAC standard was introduced in 1969 , and has been in use at DIII-D for over twenty years. Much of the CAMAC infrastructure at DIII-D is original, and has been showing signs of degradation. To make matters worse, many of the components in these systems are no longer manufactured or supported by the industry. Maintenance is becoming increasingly difficult, and the older technology falls short of meeting the capabilities of the newer computer systems that accumulate and process the data. It became evident at DIII-D (as it has at other fusion research facilities) that alternatives to CAMAC must be sought for data acquisition. A wholesale replacement of all installed CAMAC equipment at DIII-D would be cost-prohibitive. However, as needs for new data acquisition systems arise, or as individual CAMAC systems can no longer be maintained, then alternative new systems would be utilized to address these needs.

\section{REQUIREMENTS FOR DATA ACQUISITION SYSTEMS}

In order for a new data acquisition device to be efficiently utilized at DIII-D (whether as a replacement for CAMAC components or as an addition), it must be compatible with the way data is usually collected at the facility. Requirements must be met in the areas of collection, storage and upload of the data, as outlined here:

\section{A Collection}

In general, synchronous clock pulses determine the instants at which data points are acquired at DIII-D. Clock frequencies may range from a few hertz to over $1 \mathrm{MHz}$. In addition, step-wise changes in clock frequencies may occur during an acquisition sequence. Therefore, digitizers in new systems must accept external clocks whose frequencies typically change suddenly from one value to the next over a wide range of possible values. The amplitude of each data point must be recorded with 12- to 16-bit resolution.

\section{B. Storage}

Data acquired by the digitizers must be temporarily stored in data "servers" such that the data is quickly available to researchers until being archived.

\section{Upload}

Temporarily stored data must be uploaded periodically to a mass storage (archive) device. Uploads typically occur every $20 \mathrm{~min}$.

\section{ADOPTED SYSTEM STANDARD}

After assessing the product market and considering the strategies of other fusion research facilities, a system standard was proposed for new or upgraded data acquisition systems at DIII-D. The proposed system consisted of a Linux-based PCI or Compact-PCI (cPCI) computer fitted with digitizer boards. The cPCI configuration is preferred because of its greater suitability for use in the research environment: it is generally more rugged than the PCI configuration, and as with CAMAC crates, modules can be extracted and installed via front panel access, without the need to remove the entire chassis from its installed location and/or remove cabinet housings. However, where environmental conditions allow, the PCI configuration is just as suitable, and $\mathrm{PCI}$ components are usually available at 
lower cost and with more options and features. Software and programming are nearly identical for both formats.

The digitizers used for initial tests were procured from D-TACQ Solutions Ltd. These were selected because they provided the sampling speed, bit resolution, memory depth and channel count necessary to meet the specifications of several needed new data acquisition systems, and at reasonable cost. The digitizers could utilize the external clocks and triggers available at DIII-D, and had the pre- and post-trigger capture capabilities needed for most signals being acquired. D-TACQ also provided the software and drivers necessary to program and operate the digitizers from within the Linux operating system.

A typical data acquisition system was thus comprised of a computer "box" (Fig. 1) configured as a data server that could be installed near sources of data in the DIII-D facility. Analog signals to be digitized typically arrive in the vicinity of the box via shielded-twisted-pair wires or coaxial cables. These connect to patch panels with BNC, LEMO or terminal block connectors. For each digitizer board, a group of signals leaves a patch panel in a SCSI-type cable which plugs into the digitizer in the box. Clock and trigger signals also connect to each digitizer via an RJ-45 connector. A network connection to the box's Ethernet port provides a path for TCP/IP socket control, data serving and uploading.

Data acquisition during an experiment shot sequence generally proceeds as follows: As a new shot is being prepared for, the DIII-D control computer notifies the box of the current "phase" of the shot sequence. Software in the box sets acquisition parameters and appropriate states of the digitizers.
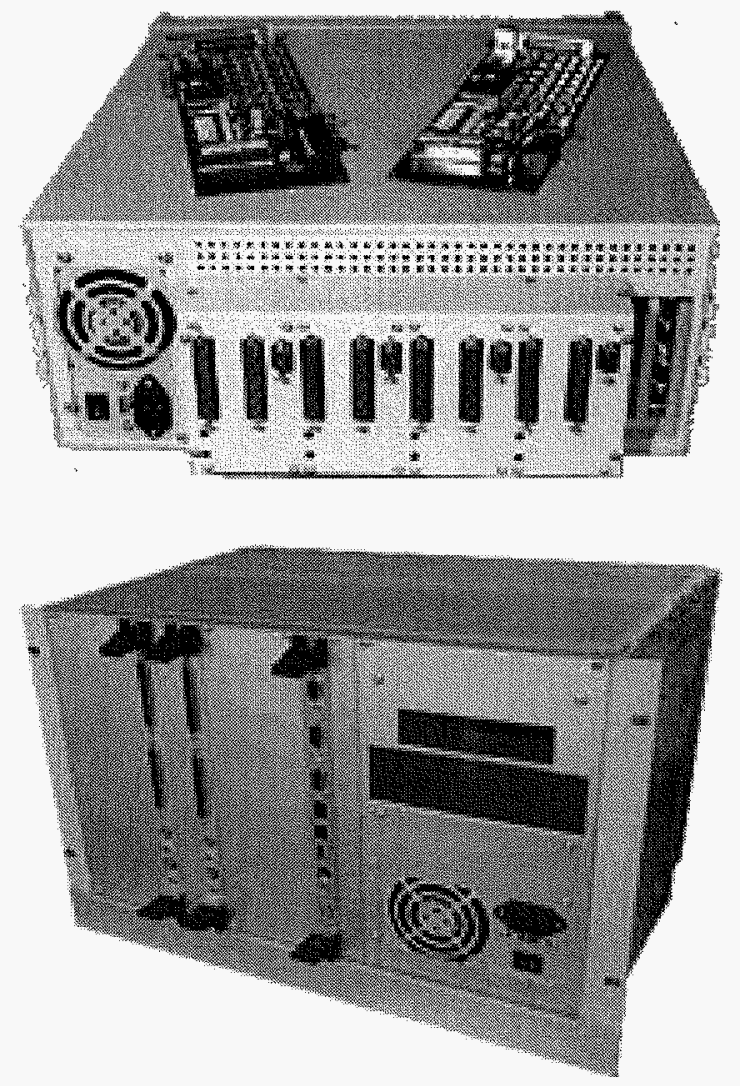

Fig. 1. PCI (top) and cPCI (bottom) data acquisition systems. (Images printed with permission of D-TACQ Solutions Ltd.)
These parameters may include the acquisition mode, the number of pre- and post-trigger samples to be acquired, the sources of clocks and triggers to be used, and the digitizer channels to enable. Before the shot begins, the digitizers are armed, and they begin acquiring and buffering data. Once the shot starts, an external trigger signal received by the digitizers initiates storage of acquired data within each digitizer's onboard memory. Data is acquired at a rate determined by the external clock, and acquisition terminates once the programmed number of pre- and post-trigger samples have been collected. Soon after the shot is complete, software in the box transfers data from digitizer memory to the local hard disk drive, where it is available for serving over the network. The entire process is then repeated for subsequent shots. Periodically (typically every $20 \mathrm{~min}$ ), software in the box compresses recent data on the hard drive and sends it over the network to the DIII-D mass storage device, from where it is subsequently served.

\section{PROTOTYPE INSTALLATIONS}

The new data acquisition system standard has been employed in four installations at DIII-D. In each case, implementation was more of a developmental process than a routine substitution; the installations functioned as "proving grounds" for the selected technologies while data acquisition needs were being met.

\section{A. Electron Cyclotron Emission Diagnostic}

The electron cyclotron emission (ECE) diagnostic measures microwave emissions to determine electron temperature in different regions of the DIII-D plasma [1]. Signals were originally acquired with CAMAC digitizers sampling at a rate of $100 \mathrm{kSps}$ (kilo-samples-per-second) with 12-bit resolution. An upgrade was requested to acquire and store up to $5 \mathrm{~s}$ of data from 40 signal channels at a new rate of $500 \mathrm{kSps}$, and with a higher resolution of 14- to 16-bits. The system needed to accept typical DIII-D clock and trigger signals, and like the CAMAC hardware it was to replace, it had to be capable of serving acquired data and uploading the data into the DIII-D mass storage device.

A new system, utilizing digitizer boards made by D-TACQ Solutions Ltd., was proposed. The boards selected were of the PCI format, and could simultaneously sample and store data from 16 channels at a maximum rate of $1.8 \mathrm{MSps}$ (mega-samples-per-second), with 14-bit resolution. External clocks and triggers could be used, and varying clock rates were acceptable. Each board also contained $128 \mathrm{MB}$ of sample memory, enough to store $8 \mathrm{~s}$ of data from all channels when a $500 \mathrm{kHz}$ clock was used.

One digitizer board was initially purchased for hardware validation experiments. The board was installed in an existing Linux-based computer (PC) at DIII-D along with the required software. The system was located near sources of signals, clocks and triggers. An existing signal feed was split and connected to both its original CAMAC digitizer and to a digitizer input on the test system. The test system was connected to the same clock and trigger signals that fed the CAMAC digitizer.

During DIII-D experiment shots, data on the signal feed was acquired by both the CAMAC and D-TACQ digitizers. After each shot, data from the CAMAC digitizer was auto- 
matically uploaded to the data acquisition computers (as usual). Data from the D-TACQ digitizer was manually uploaded to the local hard disk drive of the PC in which the digitizer was installed. Later, this data was transferred to temporary files in the acquisition computers, from where it could be plotted for comparison with the corresponding CAMAC data. Data from the D-TACQ digitizer demonstrated proper tracking as the acquisition clock stepped through frequency changes. The higher bit-resolution of the D-TACQ digitizer resulted in finer details being visible in its waveforms. It was thus demonstrated that the D-TACQ digitizers could be used in place of CAMAC digitizers with improved performance.

Since the D-TACQ digitizer had demonstrated its suitability for ECE data acquisition, a complete system was purchased, including an industrial rack-mount PC chassis, a single-board computer CPU, three 16-channel digitizers, interfacing cables and connectors, and software. Security settings were installed and an IP address was assigned to prepare the system for Ethernet connection at DIII-D. Software was written and installed to provide for operational setup and control, as well as for data serving and uploading.

One problem encountered with the new system was failure of the system to respond reliably to trigger inputs. During setup of a digitizer before an acquisition event, the number of samples to be acquired before and after receipt of a trigger can be programmed. When armed, each digitizer reads and stores data continuously into its memory in a circular buffer manner. This provides for capture of pre-trigger samples, if so requested. Once a trigger pulse is received, the digitizer continues to acquire only the additional number of post-trigger samples for which it was programmed. Acquisition then stops, and only the number of pre- and posttrigger samples requested are held in memory. The problem was that the typical $500 \mathrm{~ns}$ (nano-second) DIII-D trigger pulses were too short in duration to be reliably recognized by the digitizers. The problem was solved by passing the trigger signal through a pulse stretcher, a device capable of triggering on a very short pulse and outputting a longer one. Trigger response has been reliable ever since.

Another issue which arose was the input voltage range of the D-TACQ digitizers. The ECE signal voltage range was 0 to $+10 \mathrm{~V}$. The input voltage range of the D-TACQ digitizers, however, was -2.5 to $+2.5 \mathrm{~V}$. To prevent overloading of the digitizer inputs and to maximize resolution, it was necessary to attenuate the input signals by a factor of 2 , and to offset them by $-2.5 \mathrm{~V}$. Since a redesign of the signal amplifiers was already required for other reasons, the necessary attenuation and offset functions were incorporated in the redesign to match the input requirements of the digitizers.

\section{B. Beam Emission Spectroscopy Diagnostic}

The beam emission spectroscopy (BES) diagnostic measures Doppler-shifted light emissions from neutral beam/plasma interactions to indicate localized density fluctuations in the DIII-D plasma [2]. 32 signals were originally split and acquired with parallel "fast" and "slow" CAMAC digitizers. The fast digitizers sampled at a rate of 1.0 MSps, and their memory capacity limited acquisition to $0.5 \mathrm{~s}$. An upgrade was needed to acquire and store one second or more of data at 1.0 MSps. In addition, the CAMAC crate housing the digitizers interfaced with an older VMS-based workstation, and it was desired to replace this with a more modern Linux-based PC.

A complete PCI system (similar to the ECE system described above) was purchased from D-TACQ. This system included two 16-channel digitizers for "fast" acquisition, and one 32-channel digitizer for "slow" acquisition. The 16channel boards had the same specifications as those used for ECE: 1.8 MSps maximum sample rate, 14-bit resolution, $128 \mathrm{MB}$ of memory. The 32-channel board featured a $250 \mathrm{kSps}$ maximum sample rate, 16-bit resolution, and $128 \mathrm{MB}$ of memory. Typical sample rates used for the BES "fast" and "slow" digitizers are 1.0 MSps and $10 \mathrm{kSps}$, respectively.

The BES acquisition system utilized a trigger with much longer duration than that in the ECE system, so recognition of the trigger was not a problem. However, another issue became evident in use: the two 16-channel digitizer boards seemed to respond to the trigger at slightly different times, so that one group of 16 channels would be acquired as much as six clock pulses $(6 \mu \mathrm{s})$ earlier than the other 16 channels. The resulting skew was significant in the BES data, where inter-signal phase information is important. The situation was brought to the attention of personnel at D-TACQ, who identified and addressed the problem with updates to digitizer firmware. Great improvement was made, and data skew now occurs only rarely in triggered acquisitions.

\section{Internal Coil Instrumentation}

A new system of twelve internal coils (I-Coils) was recently installed at DIII-D for resistive wall mode feedback control and for improved error field correction [3]. A data acquisition system was needed to acquire voltage and current data for these coils and the power supplies connected to them [4]. Because the location proposed for the new acquisition system was a somewhat harsh, industrial environment, a decision was made to configure this new system in the cPCI form factor. It was initially necessary to acquire 63 signals at sample rates up to $20 \mathrm{kSps}$ and at least 10-bit resolution. A system was purchased from D-TACQ which included a cPCI computer chassis, a CPU board, and three 32-channel digitizers, each capable of acquiring data at rates up to $250 \mathrm{kSps}$ with 16-bit resolution. $128 \mathrm{MB}$ of memory on each board allowed up to $100 \mathrm{~s}$ of data to be stored from each channel with a $20 \mathrm{kHz}$ clock.

Because this was a new installation, there were no CAMAC systems nearby from which to obtain clock and trigger signals. It was necessary to run cables from existing remote sources of clock and timing signals, and a new timing receiver was installed near the system to decode trigger pulses. The receiver provided trigger pulses of sufficient width to reliably trigger the digitizers, avoiding the need for additional pulse stretchers. To protect the acquisition system from possible fault energy, isolation amplifiers were provided for the signals being digitized. The entire system was installed in an enclosed, climate controlled cabinet with temperature interlocks and a backed-up power source.

\section{Plasma Control System}

The DIII-D plasma control system (PCS) is a computerbased system used for digital control of plasma parameters. Approximately 350 analog input signals are digitized and processed by algorithm codes to generate the analog output 
signals necessary for control. Digitized signals are also stored, served and uploaded to mass storage, as with other DIII-D data acquisition equipment.

The original PCS was based on a VME computer that was connected to crates of CAMAC digitizers for data input. Several issues prompted a major upgrade of the PCS [5]. First, it was desired to expand the system beyond the limits of the original equipment. Next, more modern computers were needed to run improved control algorithms. Finally, there were benefits to relocating portions of the PCS closer to various diagnostics at DIII-D. To address these issues, the PCS was recently upgraded to a distributed system including eight $\mathrm{PCI}$ computers and one VME computer. The computers communicate with one another over a fast Myrinet network. This network also connects to a separate "gateway" computer which provides the Ethernet communication path to the PCS as a whole. Eleven 32-channel D-TACQ digitizers were installed in six of the PCI computers. The digitizers can acquire data at rates up to $250 \mathrm{kSps}$ with 16 -bit resolution. However, instead of storing data in digitizer memory, each sample of data acquired is fed directly over the PCI bus to host memory in the local CPU. From there, the data is available for real-time processing. In addition, data is processed in one of the PCI computers for real-time display on control console monitors which function much like oscilloscope displays. When the experiment shot is complete, data in each PC's memory is sent to the gateway for storage on its hard drive. The gateway serves data from the PCS and eventually transfers the data to the mass storage device.

It was important to coordinate clocking of the PCS digitizers with control cycle update times. This was accomplished with a dedicated clock distribution system. The $1 \mathrm{MHz}$ DIII-D synchronous clock is fed to one PCS digitizer designated as the "master" digitizer. The master divides the $1 \mathrm{MHz}$ down to $20 \mathrm{kHz}$, which is sent to clock all other PCS digitizers (including the master itself). Based on requirements of the processes running in each CPU, the $20 \mathrm{kHz}$ may be further divided down in the various digitizers to as low as $4 \mathrm{kHz}$ to accommodate different cycle update times. Counters keep track of the exact time each sample was acquired with respect to the $1 \mathrm{MHz}$ clock. This keeps the data used in all processes synchronized in time.

\section{OPERATIONAL EXPERIENCE AND FUTURE PLANS}

The four new data acquisition systems at DIII-D are being used regularly in support of physics experiments. Their operation has been extremely reliable, and in addition to the advantages of their higher-performance digitizers, some of the benefits of the new network configurations are already becoming evident. For example, with the CAMAC systems, many crates are connected in large, serial loop highways, each driven by an acquisition computer. Data from each channel, of each module, of each crate, is serially loaded in a prescribed order into the acquisition computer, which then serves the data. This causes long delays in the availability of some data, and big time differences between the availabilities of various data groups. With the new systems, each PC is a data server for its own digitizers. Data in digitizer memory is transferred very quickly over the PCI bus to the local hard drive, from where it is ready to be served. There is no need to wait for other systems to transfer their data first. Another advantage of the new system configuration is its overall immunity to individual failures. With a CAMAC highway system, a communication failure in one crate stops communications over the entire highway, preventing data transfer from all crates on the highway. The location of the failure is seldom evident, and time-consuming troubleshooting techniques must be employed until the source of the failure can be found and rectified. With the new system configuration, a failure in a PC affects only data from that PC. Requests for data from the faulty PC will generate error messages positively identifying that system. Data service from other PC's is unaffected.

Plans are in the works to modify or expand some of the new acquisition systems. For example, as more power supplies are brought on line to feed the I-coils, their signals will be added to that system's available digitizer channels. Expansion of the PCS will involve the addition of more networked computers and digitizers to acquire and process new data from different sources. Also, code development for the ECE system will be aimed toward sub-sampling some of the slower signals to optimize the amounts of data collected. Other new PCI or cPCI acquisition systems will inevitably be commissioned as new needs arise or as CAMAC systems fail to meet demands.

Discussions are in progress with vendors to enhance the capabilities and functions available in system modules. At present, modules primarily provide analog $\mathrm{I} / \mathrm{O}$ and some digital $\mathrm{I} / \mathrm{O}$. The hope is that modules will soon be developed to take over more of the digital $\mathrm{I} / \mathrm{O}$, clock and timing functions for which CAMAC systems are still needed. Future systems are envisioned in which all data acquisition functions can be provided from within networked $\mathrm{PCI}$ or $\mathrm{cPCI}$ computers, and legacy CAMAC systems can be retired.

\section{ACKNOWLEDGMENT}

Work supported by U.S. Department of Energy Contract No. DE-AC03-99ER54463 and Grants DE-FG03-97-ER54414 and DE-F02-89ER53296.

\section{REFERENCES}

[1] M.E. Austin, J. Lohr, "ECE radiometer upgrade on the DIII-D tokamak," Rev. Sci. Instrum., Vol. 74, p.1457 (2003).

[2] G.R. McKee, et al., "The beam emission spectroscopy diagnostic on the DIII-D tokamak," Rev. Sci. Instrum., Vol. 70, p.913 (1999).

[3] G.L. Jackson, et al., "Initial results from the new internal magnetic field coils for resistive wall mode stabilization in the DIII-D tokamak," Proc. of the 30th European Physical Society Conference on Controlled Fusion and Plasma Physics, St Petersburg, Russia, (2003), to be published in the proceedings.

[4] G.L. Campbell, et al., "Data acquisition and protection for new DIII-D in-vessel coils," these proceedings.

[5] R. Johnson, et al., "Current status of upgraded DIII-D real-time digital plasma control system," Proc. of the 4th IAEA Tech. Meeting on Control, Data Acquisition, and Remote Participation for Fusion Research, San Diego, California (2003), to be published in Fusion Eng. and Design. 